\title{
Medication Safety: Reducing Anesthesia Medication Errors and Adverse Drug Events in Dentistry Part 1
}

\author{
Daniel S. Sarasin, DDS, $†$ Jason W. Brady, DMD, $\neq \mathbb{\complement}$ and Roy L. Stevens, DDS $\mid$
}

$\dagger$ Private Practice, Oral and Maxillofacial Surgery, Cedar Rapids, Iowa $\ddagger$ Private Practice, Dental Anesthesiology, Phoenix, Arizona $₫$ Attending Faculty, Dental Anesthesiology, New York University Langone Health, New York City, New York ||Private Practice, Special Care Dentistry of Oklahoma, Oklahoma City, Oklahoma

For decades, the dental profession has provided anesthesia services in office-based, ambulatory settings to alleviate pain and anxiety, ranging from local anesthesia to general anesthesia. However, despite a reported record of safety, complications occasionally occur. Two common contributing factors to general anesthesia and sedation complications are medication errors and adverse drug events. The prevention and early detection of these complications should be of paramount importance to all dental providers who administer or otherwise use anesthesia services. Unfortunately, there is a substantial lack of literature currently available regarding medication errors and adverse drug events involving anesthesia for dentistry. As a result, the profession is forced to look to the medical literature regarding these issues not only to assess the likely severity of the problem but also to develop preventive methods specific for general anesthesia and sedation as practiced within dentistry. Part 1 of this 2-part article will illuminate the problems of medication errors and adverse drug events, primarily as documented within medicine. Part 2 will focus on how these complications affect dentistry, discuss several of the methods that medicine has implemented to manage such problems, and introduce a method for addressing these issues with the dental anesthesia medication safety paradigm.

Key Words: Medication safety; Medication errors; Adverse drug events; Dental anesthesia and sedation; Dental patient safety.

$\mathrm{C}^{\mathrm{s}}$ onsensus is lacking as to a unified definition of what constitutes a medication error. A 2010 systematic review showed 26 different definitions for the phenomenon. ${ }^{1}$ One relatively concise definition describes a medication error as "a failure in the treatment process that leads to, or has the potential to lead to, harm to the patient." ${ }^{2}$ However, a more thorough definition has been established by the United States National Coordinating Council for Medication Error Reporting and Prevention (NCC MERP), which defines a medication error as follows:

Any preventable event that may cause or lead to inappropriate medication use or patient harm while the medication is in the control of the health care professional, patient, or consumer. Such events may be related to professional practice, health care products, procedures, and systems, including pre-

Received July 15, 2019; accepted for publication July 26, 2019.

Address correspondence to Dr Daniel S. Sarasin, 835 3rd Ave SE, Cedar Rapids, IA 52403; d.sarasin@mchsi.com.

Anesth Prog 66:162-172 2019 | DOI 10.2344/anpr-66-03-10

(C) 2019 by the American Dental Society of Anesthesiology scribing, order communication, product labeling, packaging, and nomenclature, compounding, dispensing, distribution, administration, education, monitoring, and use. ${ }^{3}$

A medication error has also been defined as the selection of a correct action plan failing to be completed as intended or, alternatively, the selection of an incorrect action plan to achieve an aim. ${ }^{4}$ It has further been defined as a reduction in the probability of treatment being timely and effective or an increase in the risk of harm relating to medicines and prescribing compared with generally accepted practice. ${ }^{5}$

Medication errors have been classified by a number of methods. ${ }^{2}$ One classification method is based on the stage in which the error occurs in relation to the overall sequence of medication use (eg, prescribing, transcribing, dispensing, administration, or monitoring). Another method classifies errors according to whether they occur from mistakes made during the planning phase(s) of patient care, also known as knowledge-based or rulebased errors, or mistakes made during the execution 


\section{NCC MERP Index for Categorizing Medication Errors}

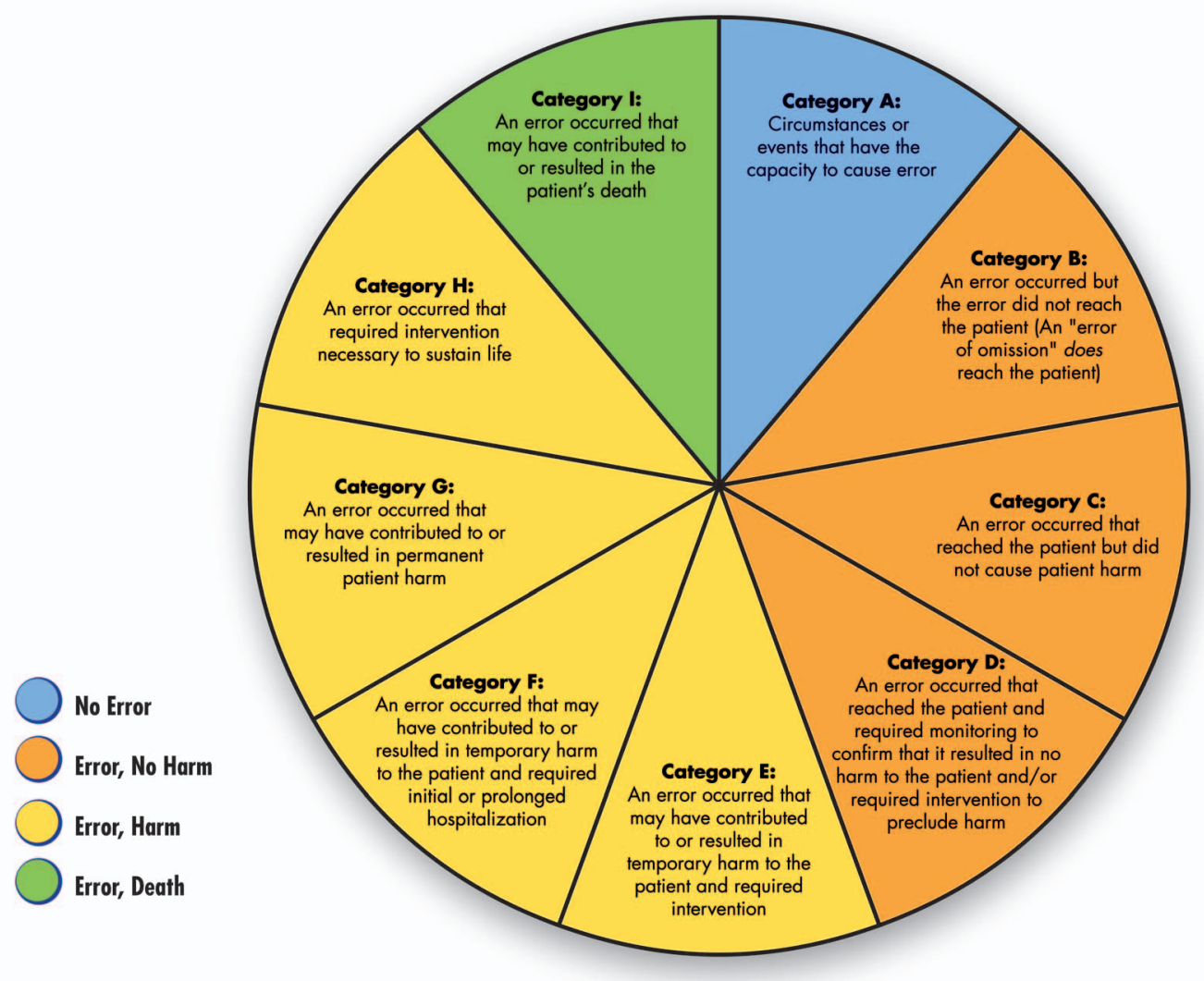

Definitions

Harm

Impairment of the physical, emotional, or psychological function or structure of the body and/or pain resulting therefrom.

Monitoring To observe or record relevant physiological or psychological signs.

Intervention May include change in therapy or active medical/surgical treatment.

Intervention

Necessary to Sustain Life

Includes cardiovascular and respiratory support (e.g., (PR, defibrillation, intubation, etc.)

๑) 2001 National Coordinating Council for Medication Error Reporting and Prevention. All Rights Reserved.

* Permission is hereby granted to reproduce information contained herein provided that such reproduction shall

not modify the text and shall include the copyright notice appearing on the pages from which it was copied.

Figure 1. NCC MERP Index.

phase(s) of care, known as "slips" (action based) or "lapses" (memory based).

Medication errors can also be classified according by their level of severity. The NCC MERP has established a Medication Error Index, which classifies medication errors based on the severity of the outcome, taking into account whether the error reached the patient and, if the patient was harmed, to what degree. ${ }^{6}$ The NCC MERP Index (Figure 1) and accompanying algorithm (Figure 2), used for determining the severity of a medication error, are used by the Institute for Safe Medication Practices reporting system, which will be discussed later in this article.

These varying approaches for classifying medication errors are not mutually exclusive, and no strong evidence supports one method of defining or classifying medication errors over another. The approach taken often will depend on the setting and the purpose for assessing such errors.

\section{DEFINING ADVERSE DRUG EVENTS}

An adverse drug event is an injury that may be predictable or unpredictable resulting from medical intervention related to a drug. ${ }^{4}$ When discussing adverse drug events and medication errors, it must be appreciated that they may be related but not necessarily codependent. An adverse drug event may or may not be due to a medication error, and a medication error may or may not cause an adverse drug event. Like medication errors, there are a plethora of classification schemes for these types of events. Some of these systems are extremely complex and classify the adverse drug event by system-organ classes and body organ groups. Edwards, et $\mathrm{al}^{7}$ described a fairly simple classification scheme that separates adverse drug events into 6 categories based on the type of reaction (Table 1).

Adverse drug events in inpatient hospital environments affect approximately 2 million hospital stays each 


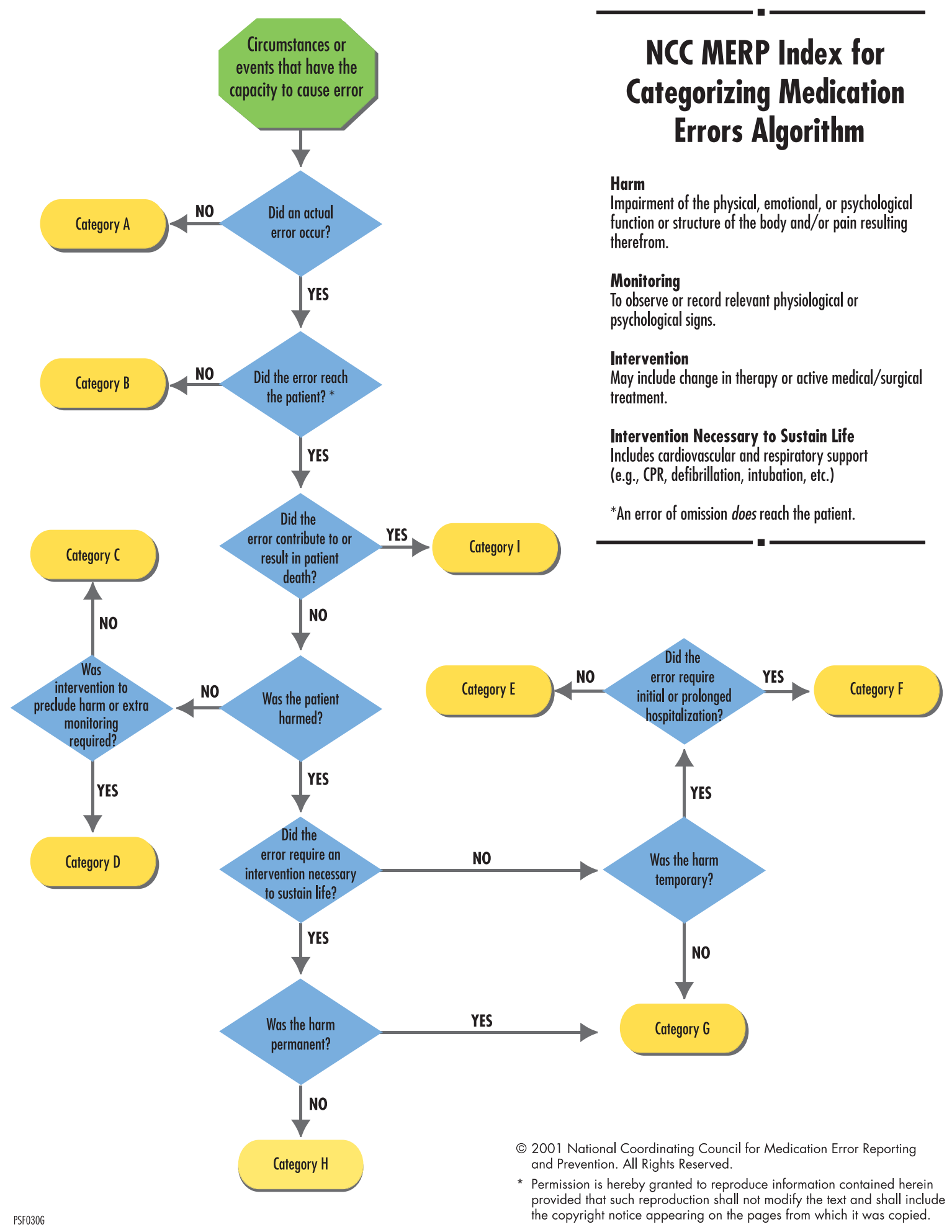

Figure 2. NCC MERP Algorithm. 
Table 1. Adverse Drug Events Classification Types ${ }^{7}$

\begin{tabular}{|c|c|c|c|c|}
\hline Type of Reaction & Mnemonic & Features & Examples & Management \\
\hline A: Dose related & Augmented & $\begin{array}{l}\text { - Common } \\
\text { - Related to drug's action } \\
\text { - Predictable } \\
\text { - Low mortality }\end{array}$ & $\begin{array}{l}\text { - Toxic effects } \\
\text { - Side effects }\end{array}$ & $\begin{array}{l}\text { - Reduce dose or withhold } \\
\text { - Consider effects of } \\
\text { concomitant therapy }\end{array}$ \\
\hline B: Nondose related & Bizarre & $\begin{array}{l}\text { - Uncommon } \\
\text { - Unrelated to drug's action } \\
\text { - Unpredictable } \\
\text { - High mortality }\end{array}$ & $\begin{array}{l}\text { - Immunologic reactions } \\
\text { - Idiosyncratic reactions }\end{array}$ & $\begin{array}{l}\text { - Withhold and avoid in } \\
\text { future }\end{array}$ \\
\hline $\begin{array}{l}\text { C: Dose related } \\
\text { and time related }\end{array}$ & Chronic & $\begin{array}{l}\text { - Uncommon } \\
\text { - Related to the cumulative } \\
\text { dose }\end{array}$ & $\begin{array}{l}\text { - Hypothalamic-pituitary- } \\
\text { adrenal axis suppression by } \\
\text { corticosteroids }\end{array}$ & $\begin{array}{l}\text { - Reduce dose or withhold } \\
\text { - Taper withdrawal }\end{array}$ \\
\hline D: Time related & Delayed & $\begin{array}{l}\text { - Uncommon } \\
\text { - Usually dose related } \\
\text { - Occurs or appears after } \\
\text { drug use }\end{array}$ & $\begin{array}{l}\text { - Teratogenesis } \\
\text { - Carcinogenesis } \\
\text { - Tardive dyskinesia }\end{array}$ & - Often intractable \\
\hline E: Withdrawal & End of use & $\begin{array}{l}\text { - Uncommon } \\
\text { - Occurs soon after drug } \\
\text { withdrawal }\end{array}$ & $\begin{array}{l}\text { - Opiate withdrawal } \\
\text { syndrome }\end{array}$ & $\begin{array}{l}\text { - Reintroduce and taper } \\
\text { withdrawal } \\
\text { - Avoid antagonist }\end{array}$ \\
\hline $\begin{array}{l}\text { F: Unexpected } \\
\text { failure of therapy }\end{array}$ & Failure & $\begin{array}{l}\text { - } \text { Common } \\
\text { - Dose related } \\
\text { - Often caused by drug } \\
\text { interactions }\end{array}$ & $\begin{array}{l}\text { - Inability to metabolize } \\
\text { codeine }\end{array}$ & $\begin{array}{l}\text { - Increased dosage } \\
\text { - Use alternative drug } \\
\text { - Consider effects of } \\
\text { concomitant therapy }\end{array}$ \\
\hline
\end{tabular}

year, prolonging those stays by an average of 1.7-4.6 days. They account for an estimated 1 in 3 of all hospital adverse events. Adverse drug events occurring in outpatient settings account for more than 1 million emergency department visits and result in more than 125,000 hospital admissions. ${ }^{8}$ An accurate assessment of the impact and prevalence of adverse drug events within the dental profession overall is currently rather difficult to ascertain because of several key factors, which will be discussed shortly. Nevertheless, the analysis, prevention, and management of adverse drug events remain as critical issues that warrant continued attention from medicine and dentistry.

\section{ASSESSING THE PROBLEM OF MEDICATION ERRORS AND ADVERSE DRUG EVENTS IN DENTISTRY}

With the exception of case reports, there is a paucity of scientific literature specifically related to the issues and incidence rates of medication errors and adverse drug events in dentistry. This is heavily due to the general nature of dentistry as well as the isolated structure of most dental practices. By and large, dentistry remains a profession that mainly uses surgical interventions, certainly to a much higher degree than strictly medical management. With a few key notable exceptions (opioids, epinephrine, local anesthetics), the risks of a medication error and/or adverse drug event occurring is likely somewhat reduced in comparison with a physician who prescribes a multitude of drugs concurrently. Dental providers who use sedation and/or general anesthesia in their practice are likely to have greater risk for medication errors and adverse drug events primarily because of the increased frequency with which drugs are being used for patient care. Unlike the traditional hospital setting, in which licensed health care professionals practice in collaborative group environments that can provide redundant decisional support, dentists often practice in a solo fashion, working essentially independently. Even when working in group practices, dentists tend to operate in working environments where they are the only licensed health care professional making individual patient care decisions. As such, dentists lack the institutional support systems inherent to medical practices in hospital environments. Simply put, there typically is not anyone else capable of "double checking" the decisions being made by the dentist. Furthermore, the vast majority of dental offices are not accredited, which is another level of safety systemically innate to medical practices in hospitals and surgery centers. Finally, state dental boards and liability insurance companies are frequently reluctant to release information that could be potentially quite useful for shedding light on and improving these areas.

Despite these obstacles, efforts are underway to improve this scarcity of data within dentistry. The Dental Patient Safety Foundation was founded in 2017 as an independent, nonprofit organization whose mission is to improve the safety and quality of dental 
care by the collecting, aggregating, analyzing, and sharing of information from dental patient safety events voluntarily and confidentially submitted by practitioners. The foundation's Patient Safety Organization status, granted by the federal government, legally protects and maintains the confidentiality of all disclosures. Practitioners are encouraged to self-report incidents through the foundation's secure website, which are then analyzed, deidentified, and published as case reports in an effort to build a database of information and improve patient safety.

The American Association of Oral and Maxillofacial Surgeons has established the Oral and Maxillofacial Surgery Quality Outcomes Registry (OMSQOR), which collects data from their members' electronic health records and uses these data to contribute to an ongoing database of oral and maxillofacial practice. An important component of OMSQOR is the Dental Anesthesia Incident Reporting System. This system is an anonymous self-reporting part of the OMSQOR used to collect and analyze anesthesia incidents in a secure and confidential manner, and it is available to all anesthesia/ sedation providers within dentistry.

The American Society of Dentist Anesthesiologists has been tracking outcomes for dentist anesthesiologists since 2010, when they joined the Society for Ambulatory Anesthesia's Clinical Outcomes Registry (SCOR), which tracked outcomes for physician and dentist anesthesiologists performing anesthesia primarily outside of the hospital, in offices and surgery centers. A dedicated data collection tool was developed for dentist anesthesiologists in SCOR, enabling them to benchmark their outcomes with other participating dentist anesthesiologists. When SCOR ceased operations, American Society of Dentist Anesthesiologists opened communications with the American Society of Anesthesiologists and the Anesthesia Quality Institute regarding participation in the National Anesthesia Clinical Outcomes Registry, the largest database of anesthesia outcomes in the United States. That relationship was solidified in April 2019, providing dentist anesthesiologists a mechanism for tracking outcomes via a formal, nationally recognized anesthesia database.

However, until these efforts mature and data can be more fully analyzed and published, the dental profession is forced to look to the medical literature as a surrogate to assess the likely prevalence and severity of medication errors and adverse drug events. Many of the preventive methods for addressing these issues developed and implemented by medicine likely have potential use within dentistry, particularly with regard to the use of general anesthesia and sedation as practiced within dentistry.

\section{IDENTIFICATION AND MANAGEMENT OF MEDICATION ERRORS AND ADVERSE DRUG EVENTS IN MEDICINE}

Medication errors and adverse drug events are key global public health concerns that have been studied extensively for more than 30 years. These issues are shared across a multitude of health care settings and many times present with staggering morbidity and mortality statistics and considerable financial impact. Numerous safety initiatives have been postulated and implemented to reduce the incidence of medication errors and adverse drug events, with many of these efforts appearing to have improved patient safety in hospitals and ambulatory settings. ${ }^{9}$ One of the early efforts was the formation of the Committee on Quality Health Care in America in 1998 by the US Institute of Medicine. Their landmark report titled To Err Is Human: Building a Safer Health System in America, released in 1999 and updated in 2006, has played a significant role in helping to shape the future of patient safety in health care. ${ }^{4,10}$ However, despite the many implemented changes and the continued attention focused on improving the delivery of health care, the issues persist. The aforementioned Institute of Medicine 2006 report found that drug-related errors are the most common medical errors, occurring at every stage from prescription through monitoring of the patient's response. Furthermore, it was estimated that an average of 1 drug error occurred per hospitalized patient per day, although not all errors led to patient harm. ${ }^{11}$

Gauging the extent to which new safety initiatives can improve patient safety first requires an accurate determination of the incidence of medication errors and adverse drug events. Various methods have been used to assess the prevalence of such events, including direct observation, chart review, and self-reporting. Unfortunately, the accuracy of these assessment tools varies greatly. This issue is clearly illustrated in a study by Flynn et al, ${ }^{12}$ in which 2557 drug doses were administered in a hospital ward. Detection of 456 medication errors occurred by direct observation, 34 by chart review, and only 1 by selfreporting. Objectively evaluating the impact of any initiative that reduces medication errors and adverse drug events is difficult because of the variation of findings, which are heavily dependent on the assessment tool used.

Many health care facilities subscribe to the MEDMARX registry of adverse drug events. The MEDMARX registry is the largest registry for adverse drug events in the United States, with more than 400 health care facilities voluntarily and anonymously reporting data, which is then analyzed and disseminated to member institutions. The types of data within the MEDMARX registry that are available for further analysis include error category (using the NCC MERP 
Index), type(s) of error, cause(s) of error, contributing factor(s), and product(s) involved. ${ }^{13}$

Despite the difficulty of objectively studying medication errors and adverse drug events, it is clear that such incidents have been reduced globally in many hospitals and outpatient clinics for a variety of reasons. Yet, with the increasing use of pharmaceutical agents throughout medicine, the issue persists. For more than 30 years, the Institute for Safe Medication Practices has been at the forefront of working to decrease medication errors and adverse drug events. ${ }^{14}$ As a result of the work by the Institute for Safe Medication Practices and the Committee on Quality Health Care in America, many facilities have instituted medication safety awareness and education programs. The rates and identifiable underlying causes of medication errors and adverse drug events are being more closely followed, with a variety of safety initiatives being developed, implemented, and monitored for effectiveness. In many facilities, the administration of drugs is now preformed only after multiple checks are completed by health care professionals. Technological safety advances including computerized physician systems with decision support, bar code-scanning devices for medication dispensing/administration, and electronic prescribing systems are being used with noted success. In addition, clinical practice agreements have been established between pharmacists and surgeons/physicians to improve medication safety by granting pharmacists the right to make decisions on initiating, altering, or discontinuing medications in accordance with established practice standards, institutional policies, or evidence-based medicine.

\section{MEDICATION ERRORS AND ADVERSE DRUG EVENTS IN ANESTHESIA}

The perioperative setting presents unique medication safety challenges not seen in many other areas in the hospital-based health care environment. Rapid administration of multiple drugs is frequently required in a highstress, fast-paced setting, which expectedly increases the risk of medication errors and/or adverse drug events. Anesthesia has seen its fair share of these types of complications often highly attributed to the hectic nature of the operating room environment. In addition, operating rooms were one of the last health care settings to undergo vigorous medication error and adverse drug event assessment. Many of the medication safety initiatives used in other areas in the hospital were either not available or deemed too burdensome for implementation in the operating room. Noted examples of such impracticalities include duplicate verification by multiple health care providers prior to any drug administration and use of clinical practice collaboration between pharmacists and anesthesiologists. Common examples of noted errors within the perioperative period include the use of a single-dose drug vial on more than 1 patient, administering a drug to the wrong patient, and failing to notice a patient's inappropriate response to a medication.

In 1978, Cooper et al ${ }^{15}$ first identified perioperative medication errors by retrospective critical-incident analysis. Historically, medication errors and adverse drug events occurring during anesthesia had been identified only through retrospective reviews and selfreporting. A Canadian closed-claims study by Orser and Byrick $^{16}$ found that $52 \%$ of adverse drug events occurring in the perioperative period were the result of medication errors. Orser et $\mathrm{al}^{17}$ published another survey study in which $85 \%$ of anesthesiologists reported at least 1 drug error or near miss in their careers.

To further quantify the problem of medication errors and adverse drug events, a landmark study in 2016 by Nanji et $\mathrm{al}^{9}$ sought to determine the perioperative incidence of medication errors and adverse drug events related to anesthesia occurring at Massachusetts General Hospital, a tertiary care hospital known throughout the world as a leader in medical safety. The study involved a randomized observational clinical trial using anesthesia providers and was backed up by a retrospective chart review, which was performed to identify events that were missed by observation. Approximately 1 in 20 medication administrations and 1 in 2 surgeries resulted in a medication error and/or an adverse drug event. More than one-third of the errors caused patient harm, while the other errors had the potential for injury. Interestingly, the study found no significant difference in medication error rates or adverse drug events for patients receiving general anesthesia versus those who received only sedation.

\section{TYPES OF MEDICATION ERRORS IN ANESTHESIA}

Perioperative medication administration is a complex process because of multitude of required steps:

1. The drug is initially ordered or requested by the anesthesia provider from the anesthesia cart or medication-dispensing system.

2. The drug is dispensed by the pharmacist or withdrawn from the dispensing system.

3. The medication is prepared for administration (ie, withdrawn from a vial into a syringe, diluted, and labeled appropriately).

4. The drug is administered to the patient.

5. The medication, dosage, and time of administration are documented in the anesthesia record. 
6. The patient is monitored for the effects of the drug (eg, vital signs, relevant laboratory studies).

7. Any unused medication is properly discarded.

In most circumstances, the anesthesia provider is responsible for many, if not all, of these steps with the potential for errors to occur at any of these stages. Errors made during the perioperative medication administration process can result in actual or potential adverse drug events with possible serious harm to the patient (Table 2).

\section{Requesting Errors}

The process begins with the anesthesia provider identifying that a particular drug is required and subsequently ordering or requesting the indicated drug for the specific patient and procedure. Planning errors can be made if the provider has an inadequate understanding of the pharmacologic properties of a medication as it relates to each patient (ie, ordering the incorrect dose or inadvertently exceeding the maximum dose) or if they request a drug that is incompatible with a patient's medical history/comorbidities, preexisting medications, or known allergies. There can be failure to realize that a drug will have a harmful interaction with other medications administered during the perioperative period. A provider may inadvertently request one drug when intending to request another. Ordering from a hospital pharmacy or using the assistance of another staff member introduces the possibility of communication errors, which can lead to the wrong drug ultimately being requested. Accidentally mixing up drugs with similar sounding names, such as dexmedetomidine and dexamethasone, can have significant potential for harming the patient.

\section{Obtaining Errors}

Failure to ensure that the proper drug is obtained can lead to medication execution errors. An anesthesia provider may inadvertently pick up the wrong vial of medication from their supply when they intended to select a different drug. Using the hospital pharmacy or the assistance of another staff member can lead to the wrong drug being obtained even when the correct drug was requested. Furthermore, failure to track expiration dates can lead to a provider using expired and possibly deteriorated drugs, which may or may not be harmful to the patient. Improper storage of anesthetic agents can lead to contamination and drug diversion. Stocking look-alike drugs has led to multiple case reports
Table 2. Common Types of Perioperative Medication Errors

\begin{tabular}{lc}
\hline & Medication Errors by Step \\
\hline Requesting & Incorrect drug requested \\
Obtaining & Miscommunication of drug request \\
& Incorrect drug selected/received \\
& Dispensing error \\
Expired/deteriorated drug error \\
Preparing & Labeling error \\
& Incorrect dosage prepared \\
Administering & Incorrect drug prepared \\
& Improper dose: overdose/underdose \\
& Incorrect timing \\
& Incorrect route \\
Recording & Inadvertent bolus \\
& Incorrect time or dosage recorded \\
Monitoring effect & Omission error \\
& Idiosyncratic reaction/allergic reaction \\
Discarding & Improper depth of sedation/anesthesia \\
\hline
\end{tabular}

involving accidental drug mix-ups, as evident by the similar packaging and colors found with some multidose vials of epinephrine and dexamethasone (Image 1).

\section{Preparing Errors}

Medication errors can occur due to incorrect preparation, including reconstitution, labeling, drug mixing, and calculation errors. Labeling errors such as a missing label on a syringe or incorrect/incomplete information on the label (eg, wrong drug, incorrect concentration, missing time of preparation and expiration on the syringe or vial) can easily lead to drugs being administered incorrectly. Such errors grant an opportunity for a provider to mix up insufficiently labeled syringes and administer the wrong drug or dose to a patient. Incompatible drugs may be combined in the same syringe or be administered in the same intravenous (IV) line, causing formation of a cloudy particulate that

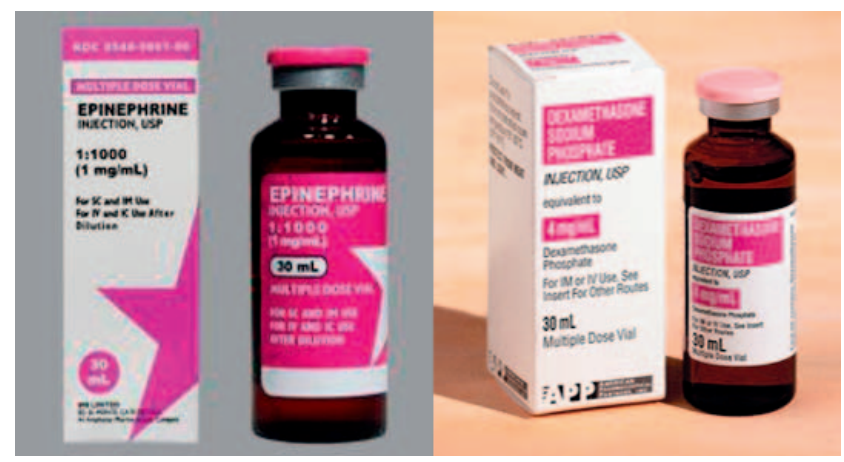

Image 1. Look-alike multi-dose vials of epinephrine and dexamethasone. 
can be deleterious to the patient's condition. Using drugs that require reconstitution or further dilution prior to administration introduces a significant risk for preparation errors, as mistakes can be made in the amount of drug withdrawn from a vial and with the amount of solution added to dilute the drug. Depending on the particular drug, the dosing error may be logarithmic in terms of scale. Providers can miscalculate the necessary dilution/reconstitution volume and prepare an incorrect final concentration or amount. Phenylephrine, usually administered in $100-$ to $200-\mu \mathrm{g}$ doses, is a classic example, as it is typically stocked as a $10-\mathrm{mg} / \mathrm{mL}, 1-\mathrm{mL}$ single-dose vial. Double dilution with $10-\mathrm{mL}$ syringes is typically required to achieve a $100-\mu \mathrm{g} /$ $\mathrm{mL}$ final concentration prior to administration.

An observational study by Avidan et $\mathrm{al}^{18}$ examined the accuracy of anesthesia faculty and residents calculating a pediatric weight-based drug dose and the corresponding amount to be drawn from a standard vial and added to a $50-\mathrm{mL}$ syringe to reach a specific infusion rate. Only $15 \%$ were correct; $61 \%$ of participants had significant calculation errors. The drug concentrations ranged from 50 times too low to 56 times too high. Interestingly, the results showed no significant difference in the presence of calculation errors between anesthesia personnel groups.

In addition, calculation errors are commonly seen in obese patients, with drug dosage being inappropriately based on actual body weight rather than ideal or lean body weight, as recommended for many anesthetic drugs. This was illustrated by Burke et $\mathrm{al}^{19}$ in 2014 during a retrospective review of anesthetic medication dosing in which they found that overweight/obese children were more likely to receive common anesthetic drugs outside the recommended dose range.

\section{Administering Errors}

Perioperative drug errors can also occur involving the actual administration of medications. Incorrect timing and/or doses can lead to overdosing or underdosing of a medication. Infusion pumps can contribute to incorrect drug delivery when the wrong administration parameters (rate, concentration, or patient information) are entered. There are many possibilities for error, such as incorrectly selecting minutes instead of hours or micrograms instead of milligrams. Users of infusion pumps can also deliver inadvertent boluses or fail to turn off the infusion when intended.

Medication errors can be caused by the drug being administered via the wrong route, such as an intramuscular drug being delivered intravenously. Similarly, the use of incorrect equipment can also lead to the wrong route of administration, potentially hindering the drug's effect. For example, epinephrine that needs to be administered intramuscularly will be inadvertently delivered into the subcutaneous tissue if the provider uses too short of a needle. Medication spillage or loss can be caused by a number of factors, such as an incompetent connection between the syringe and IV port or dislodgement of the IV cannula due to patient movement.

\section{Recording Errors}

Medication errors are occasionally the result of a failure to accurately document information regarding drug administration in the anesthesia record. Omissions and incorrectly recording time or drug information can lead to erroneously administering subsequent doses. In many environments where electronic health records and automated dispensing are not used, the anesthesia records often fail to accurately reflect the administration times. Failure to record drug administration properly can also lead to medication errors involving multiple providers, for example, both a surgeon and anesthesiologist administering antibiotics but failing to realize the error due to inaccurate recording.

\section{Monitoring Errors}

Monitoring errors are especially critical for anesthesia and sedation providers, as the types of drugs used can lead to substantial patient harm because of the potential for inhibiting normal respiratory and cardiovascular function. Improper drug management includes failure to properly monitor the patient for the effects of the drug. Failing to adequately monitor the patient's response can lead to too much or not enough of the drug's intended effect. Adverse drug events can occur when a provider fails to notice a patient's inappropriate response to a medication. Providers must be cognizant of a drug's pharmacodynamic and pharmacokinetic profile. Identification of a potent anesthetic agent's peak effect prior to discharge from the monitored perioperative environment is crucial. For example, a patient who is discharged immediately after receiving a slow-onset drug such as IV morphine has an unacceptably high risk for significant morbidity and mortality. Providers must remember that medication errors can occur even after treatment has been completed, and monitoring must continue until the patient fully meets appropriate discharge criteria. 


\section{Discarding Errors}

The failure to properly dispose of unused drugs after the procedure can lead to medication errors if a vial or syringe is left unattended. Prompt disposal of controlled substances is particularly critical as the potential for diversion and patient harm is substantial. Inappropriate disposal can increase the risk of expired/contaminated drugs being administered or a single-dose vial being reused on a second patient. Single-use vials do not contain the necessary preservatives for subsequent reuse and therefore cannot safely be used on another patient. Failure to properly discard drugs can also lead to theft by patients or staff, which may carry legal ramifications from the Drug Enforcement Agency in the case of controlled substances.

\section{CONTRIBUTING FACTORS IN MEDICATION ERRORS AND ADVERSE DRUG EVENTS}

Experts in the fields of human factor engineering, psychology, and interactive system design have identified several underlying factors related to these perioperative issues. Many of these have undergone further investigation by researchers delving into precipitating factors for medication errors and adverse drug events (Table 3 ). ${ }^{20-22}$

Interestingly, several of these factors can be directly attributed to the inherent nature of health care itself as well as design weaknesses within the organizational systems in place to support the clinician. Repetitive task design can foster reduced conscious effort, leading to boredom and increased potential for errors. Repetitive errors occurring without consequences can lead to "normalization of deviance," which describes the scenario of a clinician becoming so accustomed to an error (ie, a persistent apnea alarm) that it is no longer considered an error and is ignored. In addition, the risk for system vulnerabilities also increases with more complicated work processes requiring greater cognitive processing from the clinician. Both mental extremes, boring repetitive tasks and intense complexities, can be commonplace for anesthesia providers throughout patient care. Failure to standardize medications, chaotic/unorganized work stations, poor storage of uncommon but essential medications, and stocking similar appearing or sounding drugs (look-alike/sound-alike drugs) are several common organizational or systemic design pitfalls. Another example of a potential systemic failure is the use of a distal IV port rather than one more proximal, which increases the dead space and often becomes a forgotten drug reservoir, especially when IV flow is slowed or stopped. Accidental delivery of this drug reservoir can easily occur when flow is subsequently increased. ${ }^{23}$
Table 3. Factors that Contribute to Medication Errors and Adverse Drug Events

$$
\text { Contributing Factors }{ }^{20-22}
$$

Distraction

Haste, stress, outside pressure

Fatigue, long case times

Inadequate knowledge

Inadequate patient information

Inadequate communication

Look-alike/sound-alike drugs

Unclear/confusing labeling

New/unexpected/unfamiliar drug(s) or equipment

Complex dosing calculations

High number of drugs administered

Severity of patient's comorbidities

An additional contributing factor that reflects a systemic issue affecting all anesthesia providers is drug shortages. Medication shortages occur for a variety of reasons (drug recalls, backorders, manufacturing problems, etc), and temporary replacements may include different drug concentrations or new/unfamiliar drugs. The use of an unfamiliar drug or a different brand, packaging, or concentration of a familiar drug can increase the risk of erroneous medication administration. A classic example is ketamine, which is commonly available in varying concentrations (Image 2). One milliliter from the wrong vial could result in 10 times the intended dose and a significant potential for patient harm.

\section{CONCLUSION}

Upon reflection of the issues surrounding anesthesiarelated medication errors and adverse drug events, it becomes quite clear that the problem is multifactorial with no clear easy fix. The perioperative anesthesia
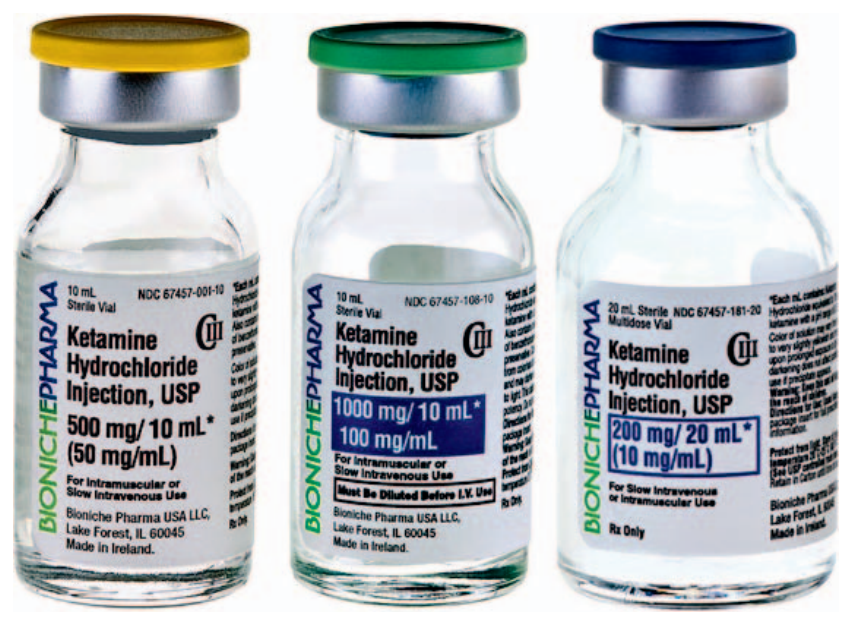

Image 2. Ketamine Vials with Varying Concentrations. 
environment is quite complex, and the administration of a multitude of potent medications is regularly required, usually with limited infrastructure incapable of completely preventing such errors. Further complicating matters are perioperative changes to the patient's condition, which may require unanticipated administration of medication resulting from the interplay of the surgical procedure, the anesthetic, and the patient's comorbidities. These alterations can occur rapidly and with fair regularity. Drug administration for anesthesia providers creates unique challenges that often directly contrast with those of other health care professionals providing care in hospital and outpatient clinical settings where established safety measures have been in place for decades.

Medication safety requires a sustained focused effort on reducing anesthesia-related medication errors and adverse drug events. An understanding of medicine's and more importantly medical anesthesia's challenges and attempts to improve medication safety is paramount prior to focusing on medication safety within anesthesia for dentistry. While the goals of improving medication safety in medicine and medical anesthesia are far from complete, dentists who provide sedation and anesthesia can learn a great deal from the trials and tribulations of their medical colleagues.

Part 2 of this article will discuss medication errors and adverse drug events as applicable to the provision of sedation and general anesthesia within dentistry, review several ways medicine and medical anesthesia have attempted to address these issues, and introduce the Dental Anesthesia Medication Safety Paradigm.

\section{REFERENCES}

1. Lisby M, Nielsen LP, Brock B, Mainz J. How are medication errors defined? A systematic literature review of definitions and characteristics. Int J Qual Health Care. 2010; 22:507-518.

2. Ferner RE, Aronson JK. Clarification of terminology in medication errors: definitions and classification. Drug Saf. 2006;29:1011-1022.

3. National Coordinating Council for Medication Error Reporting and Prevention. What is a medication error? 2015. Available at: https://www.nccmerp.org/about-medicationerrors.

4. Institute of Medicine Committee on Quality of Health Care in America; Kohn LT, Corrigan JM, Donaldson MS, editors. To Err Is Human: Building a Safer Health System. Washington, DC: National Academies Press; 2000.

5. Dean B, Barber N, Schachter M. What is a prescribing error? Qual Health Care. 2000;9:232-237.

6. National Coordinating Council for Medication Error Reporting and Prevention. Types of medication errors. 1996.
Updated February 20, 2001. Available at: https://www. nccmerp.org/types-medication-errors.

7. Edwards IR, Aronson JK. Adverse drug reactions: definitions, diagnosis, and management. Lancet. 2000;356: $1255-1259$.

8. Office of Disease Prevention and Health Promotion. Health care quality: adverse drug events: overview 2019. Updated July 22, 2019. Available at: https://health.gov/hcq/ ade.asp.

9. Nanji KC, Patel A, Shaikh S, Seger DL, Bates DW. Evaluation of perioperative medication errors and adverse drug events. Anesthesiology. 2016;124:25-34.

10. Institute of Medicine. Preventing Medication Errors. Aspden P, Wolcott J, Bootman JL, et al, eds. Washington, DC: The National Academies Press; 2007.

11. Roehr B. Institute of Medicine report strives to reduce medication errors. BMJ. 2006;333:220.

12. Flynn EA, Barker KN, Pepper GA, Bates DW, Mikeal RL. Comparison of methods for detecting medication errors in 36 hospitals and skilled-nursing facilities. Am J Health Syst Pharm. 2002;59:436-446.

13. Santell JP, Hicks RW, McMeekin J, Cousins DD. Medication errors: experience of the United States Pharmacopeia (USP) MEDMARX reporting system. J Clin Pharmacol. 2003;43:760-767.

14. Institute for Safe Medication Practices. About us. 2019. Available at: https://www.ismp.org/about.

15. Cooper JB, Newbower RS, Long CD, McPeek B. Preventable anesthesia mishaps: a study of human factors. Anesthesiology. 1978;49:399-406.

16. Orser BA, Byrick R. Anesthesia-related medication error: time to take action. Can J Anaesth. 2004;51:756-760.

17. Orser BA, Chen RJ, Yee DA. Medication errors in anesthetic practice: a survey of 687 practitioners. Can $J$ Anaesth. 2001;48:139-146.

18. Avidan A, Levin PD, Weissman C, Gozal Y. Anesthesiologists' ability in calculating weight-based concentrations for pediatric drug infusions: an observational study. J Clin Anesth. 2014;26:276-280.

19. Burke CN, Voepel-Lewis T, Wagner D, et al. A retrospective description of anesthetic medication dosing in overweight and obese children. Paediatr Anaesth. 2014;24:857-862.

20. Cooper RL, Fogarty-Mack P, Kroll HR, Barach P. Medication safety in anesthesia: epidemiology, causes, and lessons learned in achieving reliable patient outcomes. Int Anesthesiol Clin. 2019;57:78-95.

21. Gariel C, Cogniat B, Desgranges FP, Chassard D, Bouvet L. Incidence, characteristics, and predictive factors for medication errors in paediatric anaesthesia: a prospective incident monitoring study. Br J Anaesth. 2018;120:563-570.

22. Boytim J, Ulrich B. Factors contributing to perioperative medication errors: a systematic literature review. AORN J. 2018;107:91-107.

23. Kim UR, Peterfreund RA, Lovich MA. Drug infusion systems: technologies, performance, and pitfalls. Anesth Analg. 2017;124:1493-1505. 


\section{CONTINUING EDUCATION QUESTIONS}

This continuing education (CE) program is designed for dentists who desire to advance their understanding of pain and anxiety control in clinical practice. After reading the designated article, the participant should be able to evaluate and utilize the information appropriately in providing patient care.

The American Dental Society of Anesthesiology (ADSA) is accredited by the American Dental Association and Academy of General Dentistry to sponsor CE for dentists and will award CE credit for each article completed. You must answer 3 of the 4 questions correctly to receive credit.

\section{Submit your answers online at www.adsahome.org. Click on "On Demand CE."}

CE questions must be completed within 3 months and prior to the next issue.

1. As defined by the United States National Coordinating Council for Medication Error Reporting and Prevention (NCC MERP), medication errors can occur in which of the following areas?
A. Dispensing of medications
B. Package labeling
C. Prescribing of medications
D. All of the above

2. Factors that increase the risk of dental professionals being involved in medication errors and/or adverse drug events are most likely related to which of the following?
A. Dimly lit operatories and office-based settings that hinder easy visualization of medication labels
B. Federal custodial documentation that monitors supply-chain routes of controlled medications from the supplier to their administration to individual patients
C. Lack of collaborative group practice environ ments and redundant decisional support
D. Minimal length of training received during pre- or postdoctoral dental training programs in proper medication handling and dispensing

3. In a 2016 study performed at Massachusetts General Hospital, more than two-thirds of all medication errors led to permanent patient injury or harm.
A. True
B. False

4. Errors in preparation include all of the following EXCEPT:
A. Calculation
B. Disposal
C. Labeling
D. Reconstitution 